\title{
Anomalous thermodynamics of a quantum spin system with large residual entropy
}

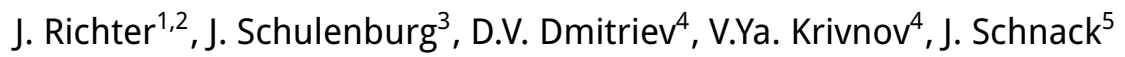 \\ 1 Institut für Physik, Otto-von-Guericke-Universität Magdeburg, P.O. Box 4120, 39016 Magdeburg, Germany \\ 2 Max-Planck-Institut für Physik komplexer Systeme, Nöthnitzer Straße 38, 01187 Dresden, Germany \\ 3 Universitätsrechenzentrum, Universität Magdeburg, D-39016 Magdeburg, Germany \\ ${ }^{4}$ Institute of Biochemical Physics of RAS, 4 Kosygin St., 119334 Moscow, Russia \\ ${ }^{5}$ Fakultät für Physik, Universität Bielefeld, Postfach 100131, 33501 Bielefeld, Germany
}

Received June 24, 2020, in final form August 14, 2020

\begin{abstract}
In contrast to strongly frustrated classical systems, their quantum counterparts typically have a non-degenerate ground state. A counterexample is the celebrated Heisenberg sawtooth spin chain with ferromagnetic zigzag bonds $J_{1}$ and competing antiferromagnetic basal bonds $J_{2}$. At a quantum phase transition point $\left|J_{2} / J_{1}\right|=1 / 2$, this model exhibits a flat one-magnon excitation band leading to a massively degenerate ground-state manifold which results in a large residual entropy. Thus, for the spin-half model, the residual entropy amounts to exactly one half of its maximum value $\lim _{T \rightarrow \infty} S(T) / N=\ln 2$. In the present paper we study in detail the role of the spin quantum number $s$ and the magnetic field $H$ in the parameter region around the transition (flat-band) point. For that we use full exact diagonalization up to $N=20$ lattice sites and the finite-temperature Lanczos method up to $N=36$ sites to calculate the density of states as well as the temperature dependence of the specific heat, the entropy and the susceptibility. The study of chain lengths up to $N=36$ allows a careful finite-size analysis. At the flat-band point we find extremely small finite-size effects for spin $s=1 / 2$, i.e., the numerical data virtually correspond to the thermodynamic limit. In all other cases the finite-size effects are still small and become visible at very low temperatures. In a sizeable parameter region around the flat-band point the former massively degenerate ground-state manifold acts as a large manifold of low-lying excitations leading to extraordinary thermodynamic properties at the transition point as well as in its vicinity such as an additional low-temperature maximum in the specific heat. Moreover, there is a very strong influence of the magnetic field on the low-temperature thermodynamics including an enhanced magnetocaloric effect.
\end{abstract}

Key words: quantum Heisenberg model, frustration, sawtooth chain, residual entropy

\section{Introduction}

The sawtooth chain is one of the paradigmatic frustrated quantum spin models built of corner-sharing triangles. The corresponding Heisenberg Hamiltonian is given by

$$
\mathcal{H}=J_{1} \sum_{\langle i, j\rangle} \mathbf{s}_{i} \cdot \mathbf{s}_{j}+J_{2} \sum_{\langle\langle i, j\rangle\rangle} \mathbf{s}_{i} \cdot \mathbf{s}_{j}-H \sum_{i} S_{i}^{z}
$$

with $\mathbf{s}_{i}^{2}=s(s+1)$. Here, the first sum runs over the zigzag bonds and the second one runs over the basal bonds, see figure 1. There are numerous studies of this spin model, see, e.g., references [1-24] within different contexts ranging from exact dimer product ground states [1, 3, 4, 14] via quantum threecoloring description [17, 18, 23] to many-body quantum scars [24]. As a prototype of a flat-band model, the sawtooth chain has attracted a particular attention by the community investigating frustrated quantum spin systems, see, e.g., references [2, 9, 10, 12, 13, 15, 16, 19, 21, 23] as well as by groups studying electronic systems, see, e.g., references [25--33], and also photonic lattices, see, e.g. references [34, 35]. 


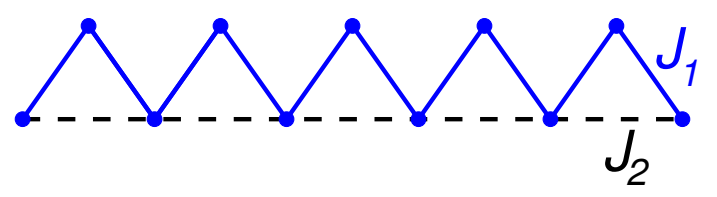

Figure 1. (Colour online) A schematic picture of the sawtooth chain.

Further motivation for theoretical studies comes from several magnetic compounds where the magnetic ions reside on sawtooth lattice sites, see, e.g. references [36-40].

The focus of the present paper is on a specific version of the sawtooth Heisenberg model with ferromagnetic (FM) zigzag bonds $J_{1}<0$ and competing antiferromagnetic (AFM) basal bonds $J_{2}>0$. We call this model the FM-AFM sawtooth chain. This model undergoes a quantum phase transition at $\kappa_{\mathrm{c}}=\left|J_{2} / J_{1}\right|=1 / 2$ from a FM to a ferrimagnetic ground state [7, 11, 15], where $\kappa_{\mathrm{c}}$ is independent of the spin quantum number $s[15]$. At the transition (flat-band) point $\kappa_{\mathrm{c}}$, the lowest one-magnon excitation band from the FM state becomes flat and it has zero energy [15, 16, 19, 22, 23]. Such a flat one-magnon band leads to a massively degenerate set of localized multi-magnon ground states resulting in an $s$-independent residual entropy $\lim _{N \rightarrow \infty} S_{0}(N) / N=\frac{1}{2} \ln 2$ [15], which is even larger than for the AFM sawtooth chain at its flat-band point [9, 10]. While the thermodynamics of the AFM sawtooth chain is well studied see, e.g., references [9, 10, 12, 13, 41], so far only a few investigations are available for the FM-AFM sawtooth chain [15, 16, 19, 22, 23]. Since the flat-band physics for this model can be observed at zero field, it might be even more interesting than the AFM model, where flat-band physics appears around the saturation field, i.e., typically at high magnetic fields. Therefore, in contrast to the FM-AFM sawtooth chain, the high-field flat-band physics for purely AFM models is not easily (or not at all) accessible in experiments, for the few exceptions see references [42-46]. A strong further motivation to extend the theoretical study of the FM-AFM sawtooth chain comes from the recently synthesized magnetic molecule $\mathrm{Gd}_{10} \mathrm{Fe}_{10}$ [38]. This magnetic system is well described by the FM-AFM sawtooth chain with of $10+10$ alternating gadolinium $\left(S=\frac{7}{2}\right)$ and iron $\left(S=\frac{5}{2}\right)$ ions, sitting on apical and basal sites, correspondingly. Importantly, the ratio of its exchange parameters is close to the transition point [16, 19, 38]. We further mention that the model is also relevant for $\mathrm{Cs}_{2} \mathrm{LiTi}_{3} \mathrm{~F}_{12}$ that hosts ferro-antiferromagnetic sawtooth chains as magnetic subsystems [47].

Here, we present a systematic study of the role of the system size $N$, the spin quantum number $s$ as well as the change of the thermodynamic properties in dependence on the distance to the transition point $d_{\mathrm{f}}=\left|J_{2} / J_{1}\right|-\kappa_{\mathrm{c}}$. Moreover, we also discuss the influence of the magnetic field on the thermodynamics, which may have a strong impact on the low-temperature physics, because it partially lifts the huge degeneracy present at $\left|J_{2} / J_{1}\right|=\kappa_{\mathrm{c}}$. To this end, we use full exact diagonalization (ED) and the finitetemperature Lanczos method (FTLM).

\section{Methods}

\subsection{Full exact diagonalization (ED)}

The exact-diagonalization technique is a powerful numerical tool which is widely applied to quantum lattice models, see, e.g. reference [48]. Using a complete set of basis states, the stationary Schrödinger equation for a finite system of $N$ sites is transformed into an eigenvalue problem. Then, the full spectrum can be determined by numerical diagonalization without approximations. We use here Jörg Schulenburg's spinpack code [49, 50] which allows one to easily treat periodic $s=1 / 2$ sawtooth chains up to $N=20$ sites.

\subsection{Finite-temperature Lanczos method (FTLM)}

The FTLM is a Monte-Carlo like extension of the full ED briefly described in the previous section. Thermodynamic quantities are determined using trace estimators [51-62]. The partition function $Z$ is 
approximated by a Monte-Carlo like representation of $Z$, i.e., the sum over a complete set of $(2 s+1)^{N}$ basis states entering $Z$ is replaced by a much smaller sum over $R$ random vectors $|v\rangle$ for each subspace $\mathcal{H}(\gamma)$ of the Hilbert space. To split the Hilbert space into small subspaces we use conservation of total $S^{z}$ as well as the lattice symmetries of the Hamiltonian, where the mutually orthogonal subspaces are labeled by $\gamma$. The exponential of the Hamiltonian is approximated by its spectral representation in a Krylov space spanned by the $N_{\mathrm{L}}$ Lanczos vectors starting from the respective random vector $|v\rangle$. Then, the FTLM approximation of the partition function is given by

$$
Z(T) \approx \sum_{\gamma=1}^{\Gamma} \frac{\operatorname{dim}(\mathcal{H}(\gamma))}{R} \sum_{v=1}^{R} \sum_{n=1}^{N_{\mathrm{L}}} \exp \left(-\frac{\epsilon_{n}^{(v)}}{k T}\right)|\langle n(v) \mid v\rangle|^{2} .
$$

Here, $|n(v)\rangle$ is the $n$-th eigenvector of $\mathcal{H}$ in the Krylov space with the corresponding energy $\epsilon_{n}^{(v)}$. As for ED, we use Schulenburg's spinpack code [49, 50] for numerical Lanczos calculations to get FTLM data for periodic sawtooth chains up to $N=36$ sites.

\section{Results}

\subsection{The model at the flat-band point}

Let us first recapitulate some important results found in reference [15]. Due to the characterization of the ground-state manifold by localized multi-magnon (LMM) states, explicit expressions were found for the degeneracies in each $S_{z}$-sector. For periodic spin-half chains with $N$ sites, the ground-state degeneracy at the transition point in a particular $S_{z}$-sector with $S^{z}=S_{\max }-k$ is

$$
\begin{aligned}
D_{N}^{k} & =\frac{n !}{(n-k) !(k) !}, \quad 0 \leqslant k \leqslant \frac{n}{2}, \quad n=\frac{N}{2}, \\
D_{N}^{k} & =\frac{n !}{(n / 2) !(n / 2) !}+\delta_{k, n}, \quad \frac{n}{2}<k \leqslant n .
\end{aligned}
$$

This yields the total degeneracy

$$
D_{N}=2^{n}+n \frac{n !}{(n / 2) !(n / 2) !}+1, \quad n=\frac{N}{2}
$$

leading to a residual entropy per site $s_{0}=\lim _{N \rightarrow \infty} \frac{1}{N} \ln D_{N}=\frac{1}{2} \ln 2$. Note that this value is independent of $s$ (for $N \rightarrow \infty$ only) and it corresponds to a system of $N / 2$ independent spin-half objects. Interestingly, the excitation gap $\Delta$ above the ground-state manifold is extremely small. Thus, for $N=20$ the ED yields $\Delta=7.502 \cdot 10^{-9}$. Moreover, $\Delta$ decreases with increasing $N$. Thus, the FM-AFM sawtooth chain at $\left|J_{2} / J_{1}\right|=\kappa_{\mathrm{c}}$ is a (rare) example of a virtually gapless finite quantum spin system.

Applying a magnetic field $H>0$, the fully polarized state with $S_{z}=S_{\max }=N s$ becomes the ground state and the former LMM ground states of the other $S_{z}$-sectors are excited states, where their excitation energy is related to the Zeeman term. Due to their huge degeneracy, see equation (3.1), this class of excitations may dominate the low-temperature physics. Thus, the contribution of the LMM states to the partition function can be explicitely given, see equation (27) in reference [15]. Based on this knowledge, universal scaling relations for the magnetization and the susceptibility were found. For the susceptibility, the universal finite-size scaling function reads [15]

$$
\chi_{N}(T)=T^{-\alpha} f\left(c_{N} N T^{\alpha-1}\right),
$$

where the $N$-dependent factor $c_{N}$ (given by a cumbersome formula) becomes $c_{N}=1 / 48$ for $N \gg 1$ and the scaling exponent $\alpha$ for $s=1 / 2$ was determined to $\alpha \simeq 1.09$ by fitting to corresponding finite-size data for $N=16$ and $N=20$. Below we verify this scaling behaviour by comparing the corresponding finite-size data for much larger systems up to $N=36$. 


\subsubsection{Density of states}

Before we present our numerical data for thermodynamic quantities such as the specific heat $c(T)$ and the susceptibility $\chi(T)$, we briefly illustrate the low-energy spectrum by discussing the excitation gaps and the density of states $\rho(E)$. In table 1 we present ED data for the excitation gaps in different sectors of $S^{z}=N s-k$ for $N=16$ and spin $s=1 / 2,1,3 / 2$ and 2. Obviously, the gaps are rather small if $k>1$, where the extreme quantum case plays a particular role with a virtually vanishing gap (see also table I in reference [15] for $s=1 / 2$ with $N=16,20,24,28$ and $k=1, \ldots, 6$ ).

In figure 2 (a) we show $\rho(E)$ for $N=16$ and spin quantum numbers $s=1 / 2$ and $s=1$. An exceptional feature of the density of states for $s=1 / 2$ is the collection of about $6 \%$ of the states in the low-energy region below $E-E_{0} \lesssim 0.6$, where this region is separated by a quasi-gap from the high-energy region $E-E_{0} \gtrsim 0.6$. This feature is also present for larger system sizes, see references [15] and [23]. The particular low-energy structure of $\rho(E)$ is important for the low-temperature physics, see below. As can be also seen in figure 2(a), the separation of the low-energy part of the spectrum is much less pronounced for $s=1$ and gradually vanishes at further increasing of $s$. Another peculiar feature of the spectrum of

Table 1. Excitation gaps $\Delta(k) \equiv E_{1}(k)-E_{0}$ of the periodic FM-AFM sawtooth chain of $N=16$ sites at the transition point $\left|J_{2} / J_{1}\right|=1 / 2=\kappa_{\mathrm{c}}, J_{1}=-1$, for spin quantum numbers $s=1 / 2,1,3 / 2$ and 2 in different subspaces $S^{z}=N s-k . E_{1}(k)$ is the energy of the lowest excitation in the subspace of $k$ magnons and $E_{0}=-12 s^{2}$ is the ground-state energy.

\begin{tabular}{|c||c|c|c|c|}
\hline & $s=1 / 2$ & $s=1$ & $s=3 / 2$ & $s=2$ \\
\hline$k$ & $\Delta(k)$ & $\Delta(k)$ & $\Delta(k)$ & $\Delta(k)$ \\
\hline 1 & 1.0 & 2.0 & 3.0 & 4.0 \\
2 & $2.178 \cdot 10^{-2}$ & $7.094 \cdot 10^{-2}$ & $8.596 \cdot 10^{-2}$ & $9.326 \cdot 10^{-2}$ \\
3 & $4.718 \cdot 10^{-4}$ & $4.829 \cdot 10^{-3}$ & $6.902 \cdot 10^{-3}$ & $7.924 \cdot 10^{-3}$ \\
4 & $9.935 \cdot 10^{-6}$ & $2.740 \cdot 10^{-4}$ & $4.797 \cdot 10^{-4}$ & $5.917 \cdot 10^{-4}$ \\
5 & $3.034 \cdot 10^{-6}$ & $1.550 \cdot 10^{-4}$ & $2.682 \cdot 10^{-4}$ & $3.248 \cdot 10^{-4}$ \\
6 & $2.584 \cdot 10^{-6}$ & $1.550 \cdot 10^{-4}$ & $2.682 \cdot 10^{-4}$ & $3.248 \cdot 10^{-4}$ \\
7 & $7.361 \cdot 10^{-7}$ & $1.550 \cdot 10^{-4}$ & $2.682 \cdot 10^{-4}$ & $3.248 \cdot 10^{-4}$ \\
\hline
\end{tabular}
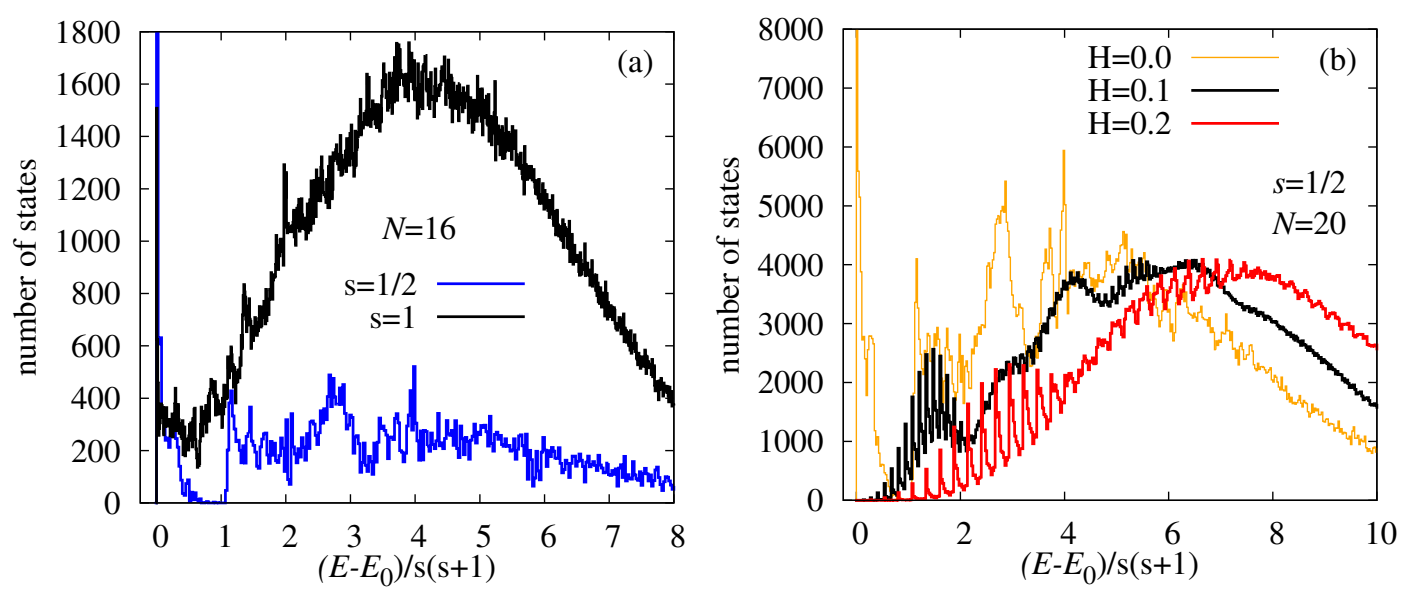

Figure 2. (Colour online) (a) Density of states (histogram, bar width $\Delta E=0.02$ ) of periodic chains of $N=16$ sites with $J_{1}=-1$ and $J_{2}=1 / 2$ for spin $s=1 / 2$ and $s=1$ (ED), where the $y$-axis is cut at 1800. Note that within the first histogram bar between $E_{0}$ and $E_{0}+\Delta E$ not only the ground states but also excited states are collected, see also table 1 (b) Field dependence of the density of states (histogram, bar width $\Delta E=0.02$ ) of periodic chains of $N=20$ sites with $J_{1}=-1, J_{2}=1 / 2$ and $\operatorname{spin} s=1 / 2(\mathrm{ED})$, where the $y$-axis is cut at 8000 . 
the $s=1 / 2$ model is the absence of the expected typical maximum of the density of states in the middle of the spectrum.

The influence of a small magnetic field on the density of states is illustrated in figure 2(b) for $s=1 / 2$ and $N=20$. As already briefly discussed above, at $H>0$, the ground state is the single fully polarized ferromagnetic state and the degeneracy of the different $S_{z}$ sectors is lifted. However, the degeneracy of the LMM states within a certain $S_{z}$ sector, see equation (3.1), is still present leading to the unconventional spiked structure of $\rho(E)$ below $\left(E-E_{0}\right) / s(s+1) \lesssim 2$ for $H=0.1$ and below $\left(E-E_{0}\right) / s(s+1) \lesssim 4$ for $H=0.2$, where the location of the peaks corresponds to the Zeeman energy of the respective $S_{z}$ sector. These parts of the spectrum related to the LMM states will dominate the low-temperature properties.

\subsubsection{Specific heat, entropy and uniform susceptibility}

Similar to the energy scale of the density of states, for the thermodynamic quantities we use the normalized temperature $T / s(s+1)$ to get a better comparison between systems of different $s$. (Note that the temperature dependences of the specific heat $c(T)$ as well as for the susceptibility $\chi(T)$ for different $s$ become identical at high temperatures as a function of $T / s(s+1)$ [63].)

In reference [15], by comparing data for $N=16,18,20,22$ for the $s=1 / 2$ sawtooth chain, it was found (i) that the low-temperature part is very specific with a long tail down to very low temperatures including two weak additional maxima below the typical main maximum and (ii) that the finite-size effects seem to be very small. It is also worth mentioning that the unconventional low-temperature part of $c(T)$ below the main maximum is entirely covered by the energy levels below the quasi-gap, cf. reference [23].

We strengthen these statements by including FTLM data up to $N=36$, see figure 3 (a), where we show the specific heat at the transition point for $\operatorname{spin} s=1 / 2$. Obviously, there are no finite-size effects down to $T / s(s+1) \sim 0.0001$ (only for the smallest system of $N=16$, we see small deviations from the curves for larger $N$ at $T<0.001)$. We also observe that the FTLM approximation is very accurate, cf. reference [60]. Thus, our finite-size data for spin $s=1 / 2$ virtually correspond to the thermodynamic limit. This feature can be attributed to the virtually vanishing excitation gaps, cf. section 3.1.1. For the temperature dependence of the susceptibility, which is related to equation (3.3), in reference [15], the formula $\chi(T)=0.317 / T^{1.09}$ was found. In figure 3 (b) we show $\ln (T \chi)$ vs. $T / s(s+1)$ for $N=16,20,24,36$. Obviously, the finite-size effects are again small and the curves for $N=24$ and 36 perfectly coincide with the above given formula for $\chi(T)$ in the whole temperature region shown in figure 3 (b). Thus, the data
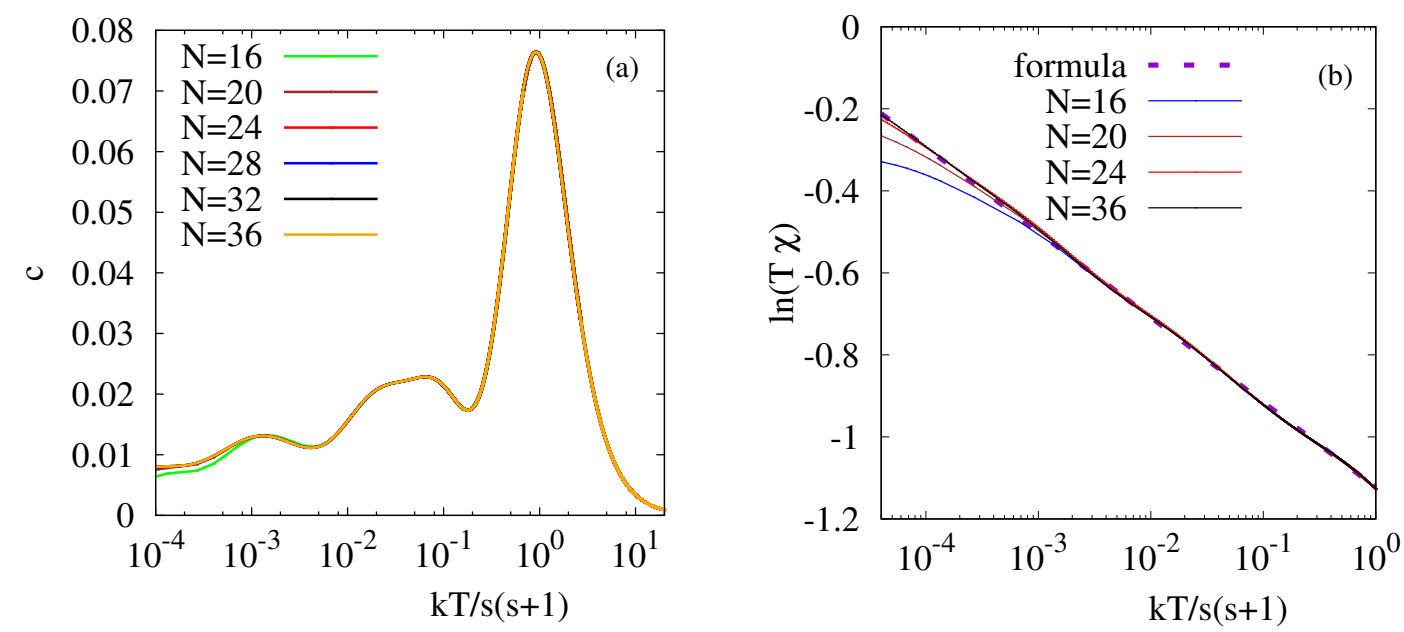

Figure 3. (Colour online) (a) Specific heat $c(T)$ per site of periodic chains of $N=16,20,24,28,32,36$ sites with $J_{1}=-1, J_{2}=1 / 2$ and spin $s=1 / 2$ (ED for $N=16,20$, FTLM for $N=24,28,32,36$ ) at zero magnetic field. (b) Log-log plot for the dependence of the susceptibility per site on temperature for periodic chains of $N=16,20(\mathrm{ED})$ and $N=24,36$ (FTLM) with $J_{1}=-1, J_{2}=1 / 2$ and spin $s=1 / 2$. The symbols correspond to the formula $\chi(T)=0.317 / T^{1.09}$. 

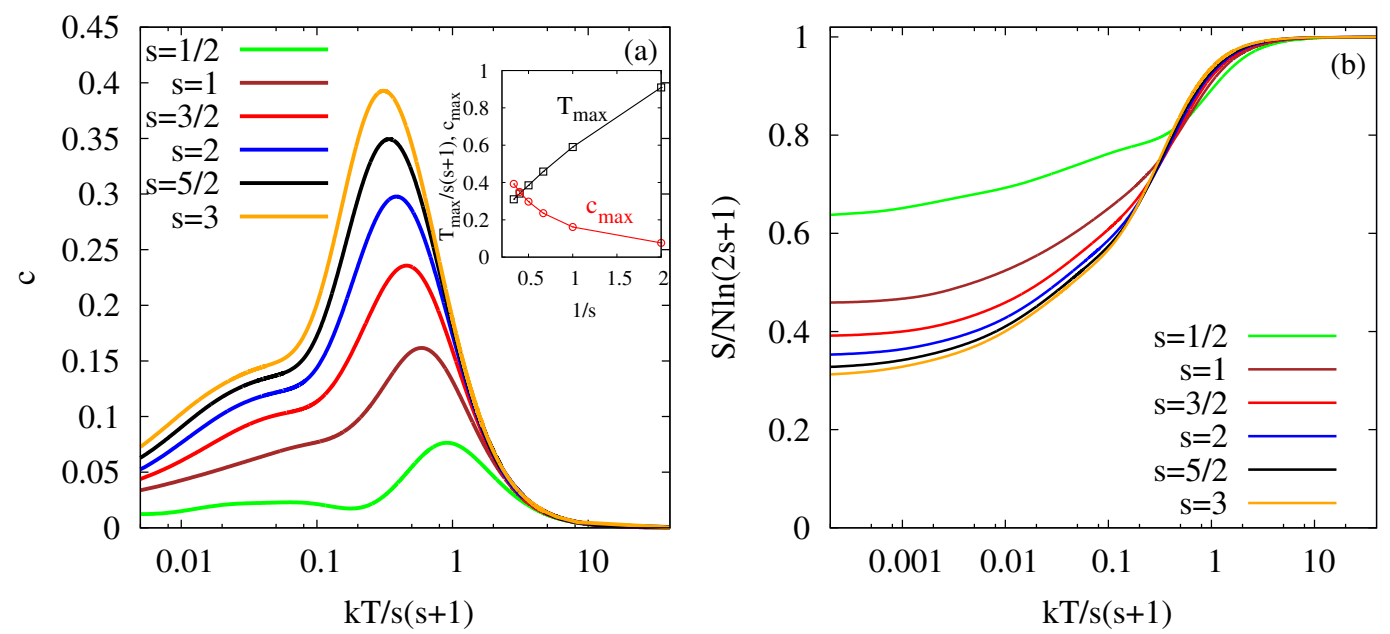

Figure 4. (Colour online) (a) Main panel: Specific heat $c(T)$ per site of periodic chains of $N=12$ sites with $J_{1}=-1, J_{2}=1 / 2$ for spin $s=1 / 2,1,3 / 2,2,5 / 2,3$ at zero magnetic field. Inset: Position $T_{\max } / s(s+1)$ and height $c_{\max }$ of the main maximum in $c(T)$ as a function of the inverse spin quantum number $s$. (b) Scaled entropy $s(T) / N \ln (2 s+1)$ per site for $N=12$ sites with $J_{1}=-1, J_{2}=1 / 2$ for spin $s=1 / 2,1,3 / 2,2,5 / 2,3$ at zero magnetic field.

for larger $N$ further confirm equation (3.3). It turns out that the scaling exponent $\alpha$ present in the scaling function equation 3.3 depends on the spin quantum number $s[22]$. It changes from $\alpha=1.09$ for the extreme quantum case $s=1 / 2$ to $\alpha=1.23$ for $s=1$ and then it smoothly tends to $\alpha=1.5$ at $s \rightarrow \infty$.

We consider now the sawtooth chain with higher spin $s$. In figure 4 (a) and (b) we show the specific heat and the entropy for $N=12$ and spin values $s=1 / 2,1,3 / 2,2,5 / 2,3$. As for $s=1 / 2$, for all $s>1 / 2$ we observe a long tail below the main maximum reaching very low temperatures. However, in contrast to the extreme quantum case $s=1 / 2$, the low-temperature part does not exhibit additional maxima, but rather there is a shoulder just below the main maximum. The position $T_{\max } / s(s+1)$ and the height $c_{\max }$ of the main maximum in $c(T)$ strongly depend on $s$, see the inset of figure 4 (a). Obviously, the maximum moves to smaller values of $T / s(s+1)$ at increasing of $s$. From the exact solution of the classical case [19] it is known that for $s \rightarrow \infty$ there is no maximum, rather the $c(T)$ exhibits a plateau-like shape with $c\left(T<T_{\mathrm{p}}\right)>0, T_{\mathrm{p}} / s(s+1) \sim 0.2$. While for the most spin systems the low- $T$ thermodynamics for the pretty large spin value $s=3$ is close to the classical case, we conclude that the highly frustrated FM-AFM sawtooth chain is an example, where $s=3$ is still far from the classical limit. For all considered values of $s$, the general entropy profiles [figure 4(b)] are similar, though with different values of the residual entropy. The slow convergence towards the classical limit with increasing of $s$ may be related to the exponentially large ground state degeneracy.

According to the larger excitation gaps for $s>1 / 2$, see table 11. we may expect that finite-size effects set in earlier as $T \rightarrow 0$. In figure 5 (a), (b), (c) we show the specific heat at the flat-band point $\kappa_{\mathrm{c}}=\left|J_{2} / J_{1}\right|=1 / 2$ for spin $s=1, s=3 / 2$ and $s=2$ and $N=12$ and $N=16$. Finite-size effects become visible below the shoulder, i.e., at about $k T / s(s+1)<0.01$, but the general shape of the $c(T)$ curve remains similar when increasing $N$.

\subsubsection{Influence of the magnetic field}

As briefly discussed for the density of states (see section 3.1.1), a magnetic field may have a drastic influence on the low-energy physics by partial lifting the massive ground-state degeneracy. Here, we focus on the specific heat $c(T)$ and the entropy $s(T)$. The magnetization process was recently studied in detail in reference [22].

We present numerical data for $s(T)$ and $c(T)$ for $N=20, N=36$ and $s=1 / 2$ in figure 6(a) and 

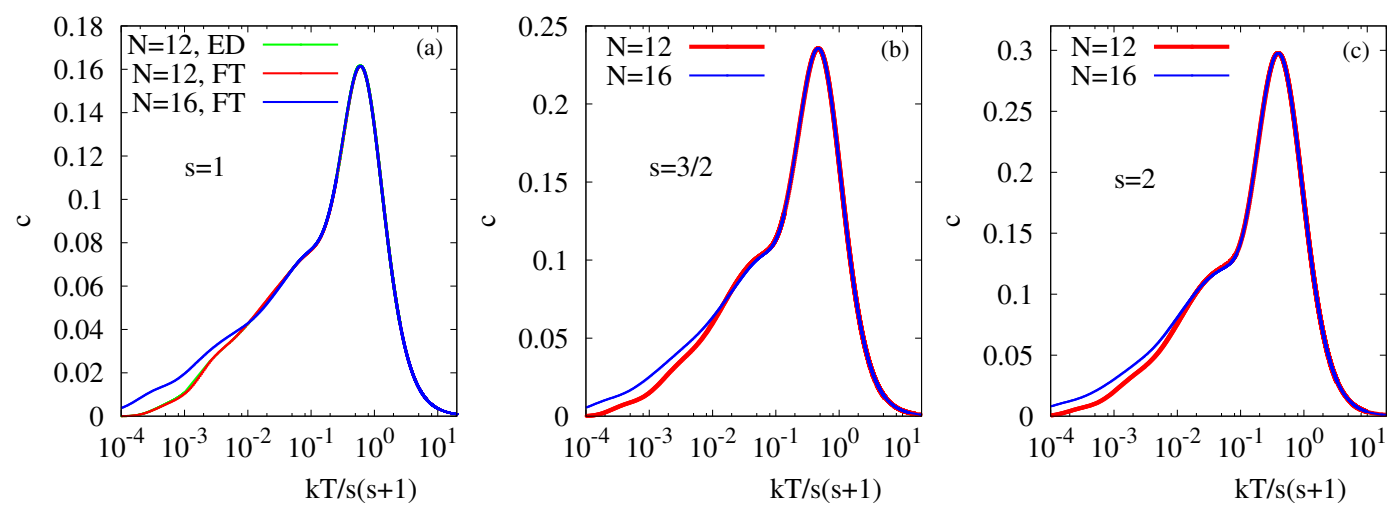

Figure 5. (Colour online) (a) Specific heat $c(T)$ per site for periodic chains of $N=12$ and 16 sites with $J_{1}=-1, J_{2}=1 / 2$ and spin $s=1$ (ED for $N=12$, FTLM for $N=12$ and $N=16$ ) at zero magnetic field. (b)-(c) Same as in (a) but without ED for $\mathrm{s}=3 / 2$ and 2, respectively.

(b), respectively, where magnetic fields $H=0,0.001,0.01,0.1,0.2$ are considered. The lifting of the massive ground-state degeneracy by the magnetic field is well visible in figure 6(a). There is no residual entropy at $H>0$, though by increasing the temperature at small fields, the entropy $s(H, T)$ pretty fast approaches the zero-field value $s(H=0, T)$ [green line in figure6(a)].

At small nonzero field $H \lesssim 0.121$, the specific heat exhibits a fairly high low-temperature maximum. This maximum is caused by a series of low-lying excitations stemming from the former degenerate LMM zero-field ground states, see the well separated sharp peaks in the density of states shown in the inset of figure 6(b). This becomes evident by the broad yellow curves which are determined using a restricted set of energies to compute the specific heat. Clearly, the position of the low-temperature maximum $T_{\max }(H)$
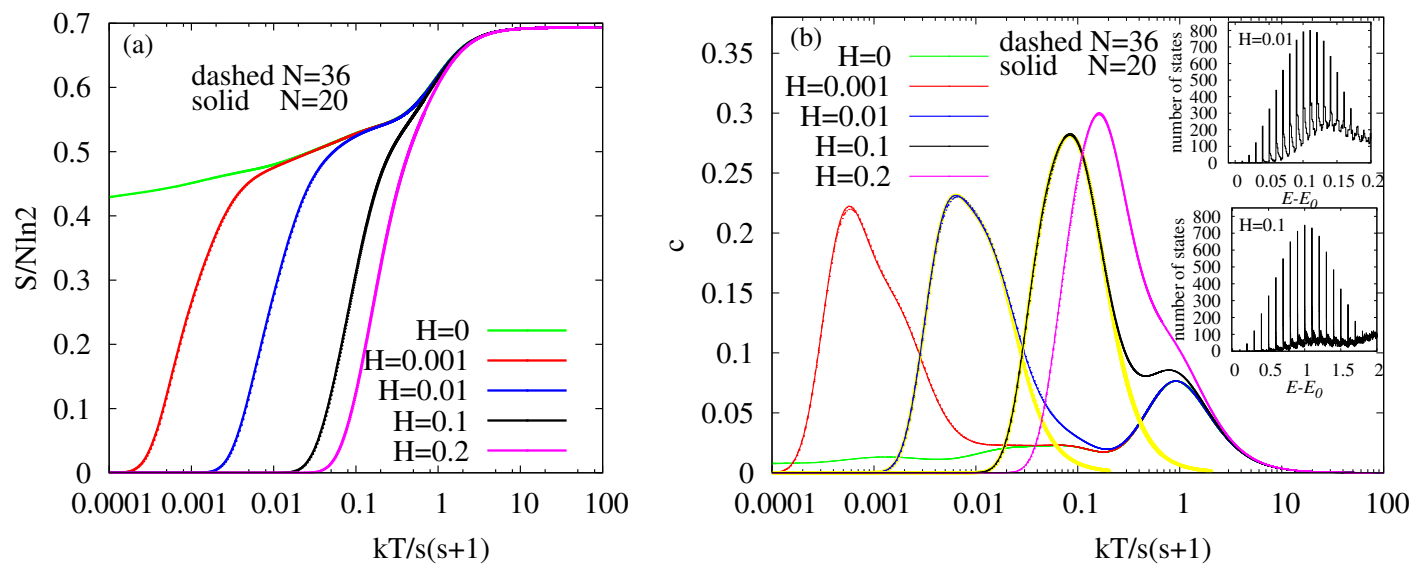

Figure 6. (Colour online) (a) Scaled entropy $s(T) / N \ln (2 s+1)$ per site for periodic chains of $N=20$ (ED) and 36 (FTLM) sites with $J_{1}=-1, J_{2}=1 / 2$ and spin $s=1 / 2$ for various magnetic fields $H$. (Note that the curves for $N=20$ and $N=36$ coincide, i.e., the dashed lines are practically not visible.) (b) Main panel: Influence of the magnetic field $H$ on the specific heat $c(T)$ per site for periodic chains of $N=20$ (ED - solid) and $N=36$ (FTLM - dashed) sites with $J_{1}=-1, J_{2}=1 / 2$ and spin $s=1 / 2$. (Note that the curves for $N=20$ and $N=36$ perfectly coincide, i.e., the dashed lines are practically not seen.) The broad yellow curves are calculated using a restricted set of energies to determine the specific heat, namely $E<E_{0}+0.15\left(E<E_{0}+1.5\right)$ for $H=0.01(H=0.1)$. Inset: Density for states for $H=0.01$ and $H=0.1$ shown for that energy region relevant for the extra low-temperature maximum in $c(T)$ presented in the main panel. 
is related to the Zeeman energies of the LMM states and it is approximately given by $T_{\max }=0.615 H$. Again, finite-size effects are negligible.

\subsubsection{Signs of flat-band physics away from the flat-band point $\kappa_{\mathrm{c}}$}

Realization of the ideal flat-band physics in an experiment on a sawtooth magnet is unlikely. Rather, one may expect that in a specific magnetic compound, the exchange parameters are sufficiently close to the flat-band point. A striking example is the FM-AFM sawtooth-chain magnetic molecule $\mathrm{Gd}_{10} \mathrm{Fe}_{10}$ [38], where the ratio of exchange parameters $J_{1}$ and $J_{2}$ is slightly below the flat-band point. However, the Gd and Fe ions carry large spins $s$ and the system of $N=20$ spins is not accessible by ED or FTLM. Hence, we focus here on spin $s=1 / 2$ that again allows the analysis of finite-size effects for $\left|J_{2} / J_{1}\right| \neq \kappa_{\mathrm{c}}$ by considering various $N$ up to $N=36$.

The LMM states stemming from the flat-band are exact eigenstates only at the flat-band point. Moving away from this point, the states and thus also the eigenvalues are modified. As a result, the massive groundstate degeneracy is lifted and the majority of the former LMM states become a large manifold of low-lying excitations. One may expect that the change of energies is smooth, where the excitation energies depend on the distance from the flat-band point $d_{\mathrm{f}}=\left|J_{2} / J_{1}\right|-\kappa_{\mathrm{c}}$.

We start with the discussion of the density of states $\rho(E)$, see figure 7, where we show the low-energy part of $\rho(E)$ for several values of $J_{2}$. The lifting of the ground-state degeneracy as well as the low-energy manifold of the former LMM ground states and their energy shift with growing $d_{\mathrm{f}}=\left|J_{2} / J_{1}\right|-\kappa_{\mathrm{c}}$ is evident. Moreover, the quasi-gap is still present and the states below the quasi-gap determine the low-temperature physics.

The specific features of the low-energy spectrum lead to a specific behaviour of the entropy shown in figure 8 . There is only a small residual entropy related to the ferromagnetic (ferrimagnetic) ground-state multiplet at $J_{2}<1 / 2\left(J_{2}>1 / 2\right)$, which vanishes as $\ln N / N$ when $N \rightarrow \infty$. This size-dependent residual entropy yields the splitting of the curves for various $N$ at low $T$. By increasing the temperature, at small deviations from the flat-band point, the entropy approaches the flat-band value $s\left(J_{2}=1 / 2, T\right)$ (blue line in figure 8 .

The specific heat $c(T)$ is shown in figure 9 (a) and (b) for a few values below and above the flat-band point $\kappa_{\mathrm{c}}$. Apparently, $c(T)$ exhibits clear signs of flat-band physics in a sizeable parameter region below and above $\kappa_{\mathrm{c}}$, namely a well-pronounced low-temperature peak coming from the former LMM ground states. On the other hand, the main peak is quite stable against small deviations from $\kappa_{\mathrm{c}}$. Noticeable finitesize effects set in around the low-temperature peak, i.e., only at very low temperatures. The position of
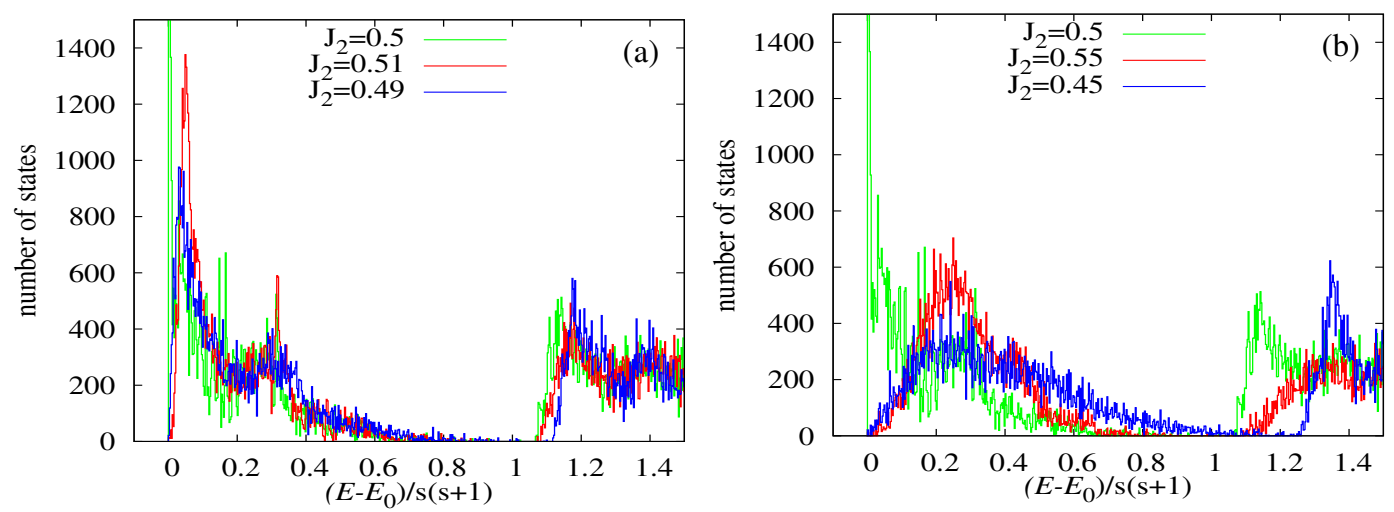

Figure 7. (Colour online) Low-energy part of the density of states (histogram, bar width $\Delta E=0.002$ ) of periodic $s=1 / 2$ chains of $N=20$ sites with $J_{1}=-1$ and (a) $J_{2}=0.49$ and $J_{2}=0.51$ as well as (b) $J_{2}=0.45$ and $J_{2}=0.55$ compared with the density of states at the flat-band point $\left(J_{2}=0.5\right)$. Note that the $y$-axis is cut at 1500 . 

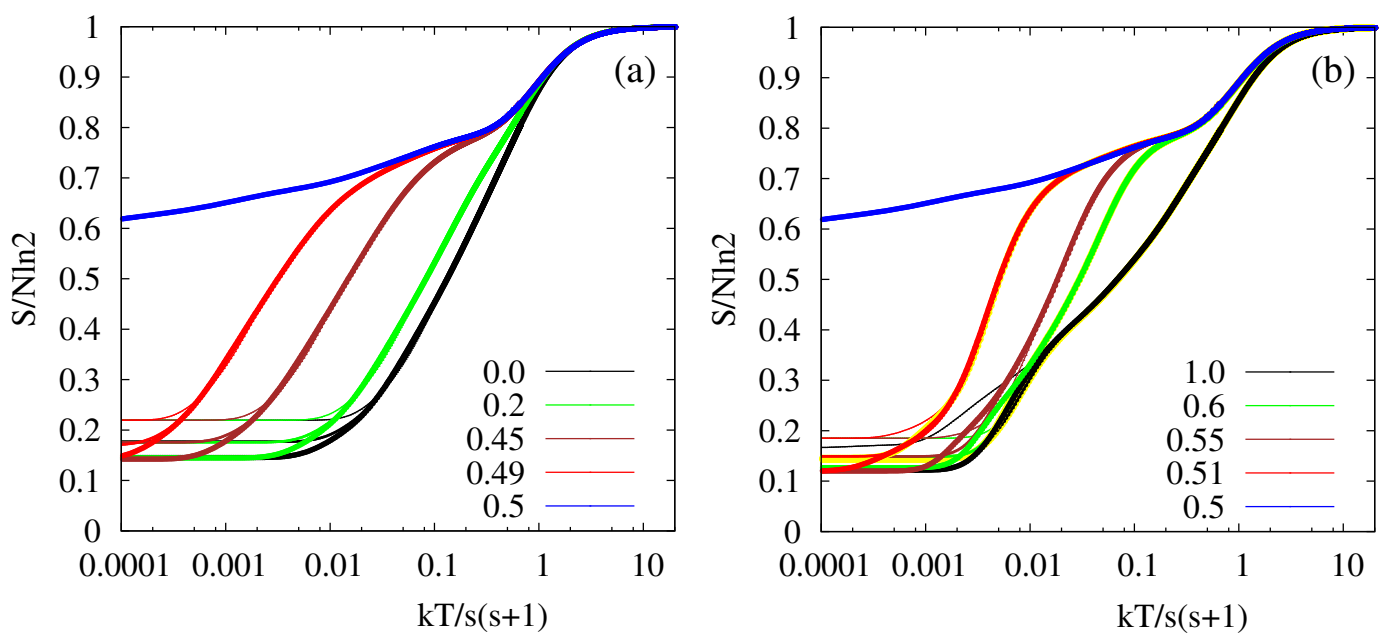

Figure 8. (Colour online) Scaled entropy $S(T) / N \ln 2$ per site of periodic chains of $N=20,28,36$ sites $[N=20(\mathrm{ED})-$ thin, $N=28(\mathrm{FTLM})-$ medium, $N=36$ (FTLM) - thick $]$ for spin $s=1 / 2, J_{1}=-1$ and various values of (a) $J_{2} \leqslant \kappa_{\mathrm{c}}$ and (b) $J_{2} \geqslant \kappa_{\mathrm{c}}$ (the $J_{2}$ values are given in the legend) at zero magnetic field.
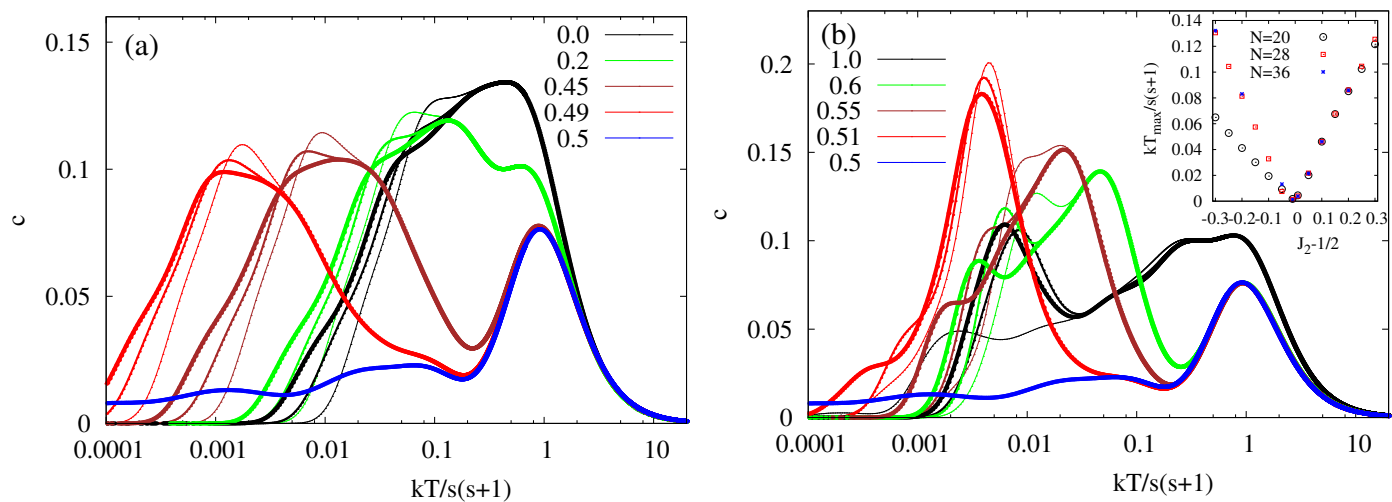

Figure 9. (Colour online) Main panel: Specific heat per site of periodic chains of $N=20,28,36$ sites $[N=20(\mathrm{ED})-$ thin, $N=28($ FTLM $)-$ medium, $N=36($ FTLM $)-$ thick $]$ for spin $s=1 / 2, J_{1}=-1$ and various values of (a) $J_{2} \leqslant \kappa_{\mathrm{c}}$ and (b) $J_{2} \geqslant \kappa_{\mathrm{c}}$ (the $J_{2}$ values are given in the legend) at zero magnetic field. The inset in panel (b) shows the position of the low-temperature maximum of $c(T)$ as a function of the distance to the transition point $d_{\mathrm{f}}=\left|J_{2} / J_{1}\right|-\kappa_{\mathrm{c}}$.

the low-temperature peak $T_{\max }$ depends on the energy-shift of the LMM states, i.e., on the distance from the flat-band point $d_{\mathrm{f}}$, see the inset in figure 9 (b). For $d_{\mathrm{f}}>0$, there is a linear relation $T_{\max } \approx 0.33 d_{\mathrm{f}}$, $0<d_{\mathrm{f}} \lesssim 0.3$, where the finite-size effects are small. On the other hand, for $d_{\mathrm{f}}<0$, the peak position is noticeably dependent on $N$, but there is no doubt of the double-maximum structure in the $c(T)$ profile.

Last but not least, we briefly discuss the magnetic cooling. There are several theoretical studies reporting an enhanced magnetocaloric effect in the vicinity of a quantum phase transition, in particular, if there is a residual entropy at the transition point, see, e.g., references [6, 9, 12, 64-66]. However, most of the previous studies in flat-band systems report on an enhanced magnetocaloric effect near the saturation field [6, 9, 12, 64, 66], which often is not accessible in experiments. By contrast, the FM-AFM sawtooth chain exhibits this phenomenon when approaching zero field [15] which is more promising while thinking in terms of a possible experimental realization. In figure 10 we show as an example 


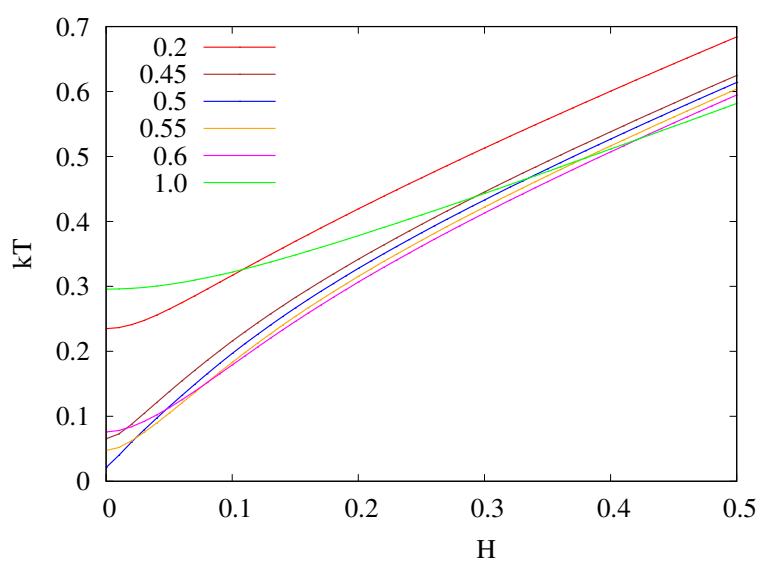

Figure 10. (Colour online) Temperature in dependence on the applied magnetic field for an isentropic cooling with fixed entropy $S / N=0.5$ for spin $s=1 / 2$ and $N=36$ and various values of $J_{2}$ (given in the legend). Corresponding data for $N=20$ (not shown) demonstrate that finite-size effects are small.

the temperature variation as a function of the applied magnetic field for an isentropic cooling with fixed entropy $S / N=0.5$ for spin $s=1 / 2$ and $N=36$ and various values of $J_{2}$. Apparently, there is a noticeable downturn in the $T(H)$ curve as $H \rightarrow 0$ for values of $J_{2}$ in the vicinity of the flat-band point, indicating the presence of an enhanced magnetocaloric effect in the FM-AFM sawtooth chain.

\section{Summary}

In the present paper we study finite spin- $s$ Heisenberg sawtooth chains with ferromagnetic (FM) zigzag bonds $J_{1}<0$ and competing antiferromagnetic (AFM) basal bonds $J_{2}>0$ by means of full exact diagonalization and the finite-temperature Lanczos method. The model exhibits, at $\kappa_{\mathrm{c}}=\left|J_{2} / J_{1}\right|=1 / 2$, a zero-temperature transition between a ferro- and a ferrimagnetic ground state. At the transition (flat-band) point $\kappa_{\mathrm{c}}$, the lowest one-magnon excitation band from the ferromagnetic state is flat (dispersionless) and has zero energy. This leads to a massively degenerate ground-state manifold resulting in a residual entropy $\lim _{N \rightarrow \infty} S_{0}(N) / N=\frac{1}{2} \ln 2$, that is independent of $s$. Moreover, in the extreme quantum case $s=1 / 2$ already for the finite systems of up to $N=36$ sites, the excitations above the ground-state manifold are virtually gapless with the result that the finite-size data practically correspond to the thermodynamic limit.

For spin quantum numbers $s>1 / 2$, the quantum effects at low temperatures remain strong, even for the largest spin $s=3$ considered here. Thus, at the flat-band point, the specific-heat profile exhibits a well-pronounced maximum with a shoulder-like part and long tail down to very low temperatures below this maximum for all $s=1, \ldots, 3$, whereas this feature is not present in the classical case.

In a sizeable parameter region around the flat-band point, the former massively degenerate groundstate manifold acts as a large manifold of low-lying excitations setting an extra low-energy scale yielding unconventional low-temperature thermodynamics which can be understood as a remnant of flat-band physics.

A specific feature of the flat-band system at hand is the strong influence of an applied magnetic field on the low-temperature properties caused by a partial lifting of degenerate ground-state manifold, i.e., most states collected in the residual entropy at zero field become low-energy excitations according to their Zeeman energy.

Finally, we argue that our results for the FM-AFM Heisenberg sawtooth chain might be (at least to some extent) representative for other systems with a large residual entropy such as the three-coloring $X X Z$ sawtooth chain [17, 18, 23] and the FM-AFM kagome chain [67]. 


\section{Acknowledgements}

JR is deeply indebted to Oleg Derzhko for many inspiring scientific discussions and a very fruitful collaboration over more than 20 years. All authors thank Oleg Derzhko for useful discussions and Andreas Honecker for critical comments. J.R. and J.S. thank the DFG for financial support (grants RI 615/25-1 and SCHN 615/28-1).

\section{References}

1. Sen D., Shastry B.S., Walstedt R.E., Cava R., Phys. Rev. B, 1996, 53, 6401, doi 10.1103/PhysRevB.53.6401

2. Schulenburg J., Honecker A., Schnack J., Richter J., Schmidt H.-J., Phys. Rev. Lett., 2002, 88, 167207, doi $10.1103 /$ PhysRevLett.88.167207

3. Chen S., Büttner H., Voit J., Phys. Rev. B, 2003, 67, 054412, doi 10.1103/PhysRevB.67.054412

4. Blundell S.A., Núñez-Regueiro M.D., Eur. Phys. J. B, 2003, 31, 453, doi:10.1140/epjb/e2003-00054-2

5. Blundell S.A., Núñez-Regueiro M.D., J. Phys.: Condens. Matter, 2004, 16, S791, doi $10.1088 / 0953-8984 / 16 / 11 / 031$

6. Zhitomirsky M.E., Honecker A., J. Stat. Mech.: Theory Exp., 2004, 2004, P07012, doi $10.1088 / 1742-5468 / 2004 / 07 / \mathrm{P} 07012$

7. Tonegawa T., Kaburagi M., J. Magn. Magn. Mater., 2004, 272-276, 898, doi 10.1016/j.jmmm.2003.11.367

8. Chandra V.R., Sen D., Ivanov N.B., Richter J., Phys. Rev. B, 2004, 69, 214406, doi 10.1103/PhysRevB.69.214406

9. Zhitomirsky M.E., Tsunetsugu H., Phys. Rev. B, 2004, 70, 100403, doi 10.1103/PhysRevB.70.100403

10. Derzhko O., Richter J., Phys. Rev. B, 2004, 70, 104415, doi:10.1103/PhysRevB.70.104415

11. Kaburagi M., Tonegawa T., Kang M., J. Appl. Phys., 2005, 97, 10B306, doi: $10.1063 / 1.1851893$

12. Derzhko O., Richter J., Eur. Phys. J. B, 2006, 52, 23, doi:10.1140/epjb/e2006-00273-y

13. Richter J., Derzhko O., Honecker A., Int. J. Mod. Phys. B, 2008, 22, 4418, doi $10.1142 /$ S0217979208050176.

14. Hao Z., Wan Y., Rousochatzakis I., Wildeboer J., Seidel A., Mila F., Tchernyshyov O., Phys. Rev. B, 2011, 84, 094452, doi:10.1103/PhysRevB.84.094452.

15. Krivnov V.Ya., Dmitriev D.V., Nishimoto S., Drechsler S.-L., Richter J., Phys. Rev. B, 2014, 90, 014441, doi $10.1103 /$ PhysRevB.90.014441.

16. Dmitriev D.V., Krivnov V.Ya., Phys. Rev. B, 2015, 92, 184422, doi 10.1103/PhysRevB.92.184422

17. Changlani H.J., Kochkov D., Kumar K., Clark B.K., Fradkin E., Phys. Rev. Lett., 2018, 120, 117202 , doi $10.1103 /$ PhysRevLett.120.117202

18. Changlani H.J., Pujari S., Chung C.-M., Clark B.K., Phys. Rev. B, 2019, 99, 104433, doi 10.1103/PhysRevB.99.104433.

19. Dmitriev D.V., Krivnov V.Ya., Richter J., Schnack J., Phys. Rev. B, 2019, 99, 094410, doi $10.1103 /$ PhysRevB.99.094410

20. Yamaguchi T., Drechsler S.-L., Ohta Y., Nishimoto S., Phys. Rev. B, 2020, 101, 104407, doi 10.1103/PhysRevB.101.104407.

21. Metavitsiadis A., Psaroudaki C., Brenig W., Phys. Rev. B, 2020, 101, 235143, doi $10.1103 /$ PhysRevB.101.235143.

22. Dmitriev D.V., Krivnov V.Ya., Schnack J., Richter J., Phys. Rev. B, 2020, 101, 054427, doi $10.1103 /$ PhysRevB.101.054427.

23. Derzhko O., Schnack J., Dmitriev D.V., Krivnov V.Ya., Richter J., Eur. Phys. J. B, 2020, 93, 161, doi $10.1140 /$ epjb/e2020-10224-1

24. McClarty P.A., Haque M., Sen A., Richter J., Phys. Rev. B, 2020, 102, 224303, doi $10.1103 /$ PhysRevB.102.224303.

25. Tasaki H., Phys. Rev. Lett., 1992, 69, 1608, doi:10.1103/PhysRevLett.69.1608.

26. Mielke A., Tasaki H., Commun. Math. Phys., 1993, 158, 341, doi 10.1007/BF02108079.

27. Tasaki H., J. Stat. Phys., 1996, 84, 535, doi $10.1007 / B F 02179652$

28. Watanabe Y., Miyashita S., J. Phys. Soc. Jpn., 1997, 66, 2123, doi $10.1143 / J P S J .66 .2123$

29. Arita R., Shimoi Y., Kuroki K., Aoki H., Phys. Rev. B, 1998, 57, 10609, doi:10.1103/PhysRevB.57.10609.

30. Derzhko O., Richter J., Honecker A., Maksymenko M., Moessner R., Phys. Rev. B, 2010, 81, 014421, doi 10.1103/PhysRevB.81.014421.

31. Maksymenko M., Honecker A., Moessner R., Richter J., Derzhko O., Phys. Rev. Lett., 2012, 109, 096404, doi 10.1103/PhysRevLett.109.096404 
32. Flach S., Leykam D., Bodyfelt J.D., Matthies P., Desyatnikov A.S., EPL, 2014, 105, 30001, doi $10.1209 / 0295-5075 / 105 / 30001$

33. Liu R., Nie W., Zhang W., Sci. Bull., 2019, 64, 1490, doi:10.1016/j.scib.2019.08.013.

34. Weimann S., Morales-Inostroza L., Real B., Cantillano C., Szameit A., Vicencio R.A., Opt. Lett., 2016, 41, 2414, doi $10.1364 /$ OL.41.002414.

35. Tang L., Song D., Xia S., Xia S., Ma J., Yan W., Hu Y., Xu J., Leykam D., Chen Z., Nanophotonics, 2020, 9, 1161, doi:10.1515/nanoph-2020-0043

36. Ruiz-Pérez C., Hernández-Molina M., Lorenzo-Luis P., Lloret F., Cano J., Julve M., Inorg. Chem., 2000, 39, 3845, doi: $10.1021 / \mathrm{ic} 000314 \mathrm{n}$

37. Inagaki Y., Narumi Y., Kindo K., Kikuchi H., Kamikawa T., Kunimoto T., Okubo S., Ohta H., Saito T., Azuma M., Takano M., Nojiri H., Kaburagi M., Tonegawa T., J. Phys. Soc. Jpn., 2005, 74, 2831, doi 10.1143/JPSJ.74.2831

38. Baniodeh A., Magnani N., Lan Y., Buth G., Anson C.E., Richter J., Affronte M., Schnack J., Powell A.K., npj Quantum Mater., 2018, 3, 10, doi 10.1038/s41535-018-0082-7

39. Heinze L., Jeschke H., Metavitsiadis A., Reehuis M., Feyerherm R., Hoffmann J.-U., Wolter A.U.B., Ding X., Zapf V., Moya C.C., Weickert F., Jaime M., Rule K.C., Menzel D., Valentí R., Brenig W., Süllow S., Preprint arXiv:1904.07820, 2019.

40. Nhalil H., Baral R., Khamala B.O., Cosio A., Singamaneni S.R., Fitta M., Antonio D., Gofryk K., Zope R.R., Baruah T., Saparov B., Nair H.S., Phys. Rev. B, 2019, 99, 184434, doi 10.1103/PhysRevB.99.184434

41. Derzhko O., Richter J., Maksymenko M., Int. J. Mod. Phys. B, 2015, 29, 1530007, doi $10.1142 /$ S0217979215300078

42. Kikuchi H., Fujii Y., Chiba M., Mitsudo S., Idehara T., Tonegawa T., Okamoto K., Sakai T., Kuwai T., Ohta H., Phys. Rev. Lett., 2005, 94, 227201, doi 10.1103/PhysRevLett.94.227201

43. Honecker A., Hu S., Peters R., Richter J., J. Phys.: Condens. Matter, 2011, 23, 164211, doi $10.1088 / 0953-8984 / 23 / 16 / 164211$

44. Okuma R., Nakamura D., Okubo T., Miyake A., Matsuo A., Kindo K., Tokunaga M., Kawashima N., Takeyama S., Hiroi Z., Nat. Commun., 2019, 10, 1229, doi 10.1038/s41467-019-09063-7

45. Tanaka H., Kurita N., Okada M., Kunihiro E., Shirata Y., Fujii K., Uekusa H., Matsuo A., Kindo K., Nojiri H., J. Phys. Soc. Jpn., 2014, 83, 103701, doi:10.7566/JPSJ.83.103701.

46. Richter J., Krupnitska O., Baliha V., Krokhmalskii T., Derzhko O., Phys. Rev. B, 2018, 97, 024405, doi 10.1103/PhysRevB.97.024405.

47. Shirakami R., Ueda H., Jeschke H.O., Nakano H., Kobayashi S., Matsuo A., Sakai T., Katayama N., Sawa H., Kindo K., Michioka C., Yoshimura K., Phys. Rev. B, 2019, 100, 174401, doi 10.1103/PhysRevB.100.174401.

48. Läuchli A.M., In: Introduction to Frustrated Magnetism, Mila F., Lacroix C., Mendels P. (Eds.), Springer, Berlin, Heidelberg, 2011, 34.

49. Schulenburg J., Spinpack-2.59, Magdeburg University, 2019.

50. Richter J., Schulenburg J., Eur. Phys. J. B, 2010, 73, 117, doi:10.1140/epjb/e2009-00400-4

51. Jaklič J., Prelovšek P., Phys. Rev. B, 1994, 49, 5065, doi 10.1103/PhysRevB.49.5065

52. Jaklič J., Prelovšek P., Adv. Phys., 2000, 49, 1, doi 10.1080/000187300243381.

53. Hams A., De Raedt H., Phys. Rev. E, 2000, 62, 4365, doi:10.1103/PhysRevE.62.4365.

54. Schnack J., Wendland O., Eur. Phys. J. B, 2010, 78, 535, doi $10.1140 /$ epjb/e2010-10713-8.

55. Prelovšek P., Bonča J., In: Strongly Correlated Systems: Numerical Methods, Avella A., Mancini F. (Eds.), Springer, Berlin, Heidelberg, 2013, 1-30.

56. Hanebaum O., Schnack J., Eur. Phys. J. B, 2014, 87, 194, doi:10.1140/epjb/e2014-50360-5

57. Schmidt B., Thalmeier P., Phys. Rep., 2017, 703, 1, doi:10.1016/j.physrep.2017.06.004.

58. Prelovšek P., In: The Physics of Correlated Insulators, Metals, and Superconductors, Pavarini E., Koch E., Scalettar R., Martin R.M. (Eds.), Forschungszentrum Jülich GmbH, Jülich, 2017, 7.1-7.23.

59. Schnack J., Schulenburg J., Richter J., Phys. Rev. B, 2018, 98, 094423, doi 10.1103/PhysRevB.98.094423

60. Schnack J., Richter J., Steinigeweg R., Phys. Rev. Res., 2020, 2, 013186, doi $10.1103 /$ PhysRevResearch.2.013186

61. Seki K., Yunoki S., Phys. Rev. B, 2020, 101, 235115, doi 10.1103/PhysRevB.101.235115

62. Schnack J., Schulenburg J., Honecker A., Richter J., Phys. Rev. Lett., 2020, 125, 117207, doi $10.1103 /$ PhysRevLett.125.117207

63. Lohmann A., Schmidt H.-J., Richter J., Phys. Rev. B, 2014, 89, 014415, doi 10.1103/PhysRevB.89.014415

64. Zhitomirsky M.E., Phys. Rev. B, 2003, 67, 104421, doi 10.1103/PhysRevB.67.104421.

65. Schnack J., Schmidt R., Richter J., Phys. Rev. B, 2007, 76, 054413, doi 10.1103/PhysRevB.76.054413

66. Wolf B., Honecker A., Hofstetter W., Tutsch U., Lang M., Int. J. Mod. Phys. B, 2014, 28, 1430017, doi $10.1142 / \mathrm{S} 0217979214300175$.

67. Dmitriev D.V., Krivnov V.Ya., J. Phys.: Condens. Matter, 2017, 29, 215801, doi $10.1088 / 1361-648 X / a a 68 f 6$. 


\title{
Аномальна термодинаміка квантової спінової системи 3 великою залишковою ентропією
}

\author{
Й. Ріхтер 112, Й. Шуленбург ${ }^{3}$, Д. В. Дмітрієв ${ }^{4}$, В. Я. Крівнов ${ }^{4}$, Ю. Шнак ${ }^{5}$ \\ 1 Інститут фізики, Університет Магдебурга, поштова скринька 4120, Магдебург 39016, Німеччина \\ 2 Інститут Макса Планка фізики складних систем, Ньотнітцерштрасе 38, D-01187 Дрезден, Німеччина \\ 3 Університетський обчислювальний центр, Університет Магдебурга, поштова скринька 4120, Магдебург \\ 39016, Німеччина \\ ${ }^{4}$ Інститут біохімічної фізики РАН, вул. Косигіна 4, Москва 119334, Росія \\ 5 Університет Білєфельда, факультет фізики, поштова скринька 100131, D-33501 Білєфельд, Німеччина
}

\begin{abstract}
На відміну від класичних сильно фрустрованих систем, їхні квантові відповідники зазвичай мають невироджений основний стан. Контрприкладом $є$ знаменитий спіновий пилкоподібний ланцюжок Гайзенберґа з феромагнітними зигзаг зв'язками $J_{1}$ і конкуруючими антиферомагнітними базисними зв'язками $J_{2}$. У точці квантового фазового переходу $\left|J_{2} / J_{1}\right|=1 / 2$ така модель виявляє плоску зону одномагнонних збуджень, яка веде до сильно виродженого многовиду основних станів, що призводить до великої залишкової ентропії. Отже, для спін-1/2 моделі залишкова ентропія становить точно половину від їі максимального значення $\lim _{T \rightarrow \infty} S(T) / N=\ln 2$. в даній роботі ми детально вивчаємо роль спінового квантового числа $s$ і магнітного поля $H$ в області параметрів біля точки переходу (плоска зона). Для цього ми використовуємо повну діагоналізацію до $N=20$ вузлів ґратки, а також скінченно-температурний метод Ланцоша до $N=36$ вузлів, щоб обчислити густину станів, а також температурну залежність теплоємності, ентропії та сприйнятливості. Дослідження ланцюжків довжиною до $N=36$ дозволяє акуратний скінченно-розмірний аналіз. В плоскозонній точці ми знайшли надзвичайно малий скінченно-розмірний ефект для спіна $s=1 / 2$, тобто числові дані віртуально відповідають термодинамічній границі. В усіх інших випадках скінченно-розмірні ефекти $€$ все ще малі і стають помітними при дуже низьких температурах. У значній області параметрів навколо плоскозонної точки вплив попереднього сильно виродженого многовиду основних станів діє як великий многовид низькоенергетичних збуджень, який в точці переходу, а також в її околі, веде до незвичайних термодинамічних властивостей, таких як додатковий низькотемпературний максимум у теплоємності. До того ж, існує дуже сильний вплив магнітного поля на низькотемпературну термодинаміку, включаючи посилений магнетокалоричний ефект.
\end{abstract}

Ключові слова: квантова модель Гайзенберґа, фрустрація, пилкоподібний ланцюжок, залишкова ентропія 


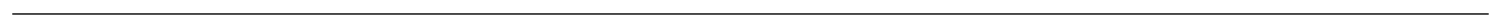

